\title{
Dilatancy of Shear Transformations in a Colloidal Glass
}

\author{
Y. Z. Lu, ${ }^{1, *}$ M. Q. Jiang, ${ }^{2,3, \dagger}$ X. Lu, ${ }^{1}$ Z. X. Qin, ${ }^{1}$ Y. J. Huang, ${ }^{4, *}$ and J. Shen ${ }^{5}$ \\ ${ }^{1}$ School of Materials Science and Engineering, Dalian Jiaotong University, \\ Dalian 116028, People's Republic of China \\ ${ }^{2}$ State Key Laboratory of Nonlinear Mechanics, Institute of Mechanics, Chinese Academy of Sciences, \\ Beijing 100190, People's Republic of China \\ ${ }^{3}$ School of Engineering Science, University of Chinese Academy of Sciences, \\ Beijing 100049, People's Republic of China \\ ${ }^{4}$ School of Materials Science and Engineering, Harbin Institute of Technology, \\ Harbin 150001, People's Republic of China \\ ${ }^{5}$ School of Materials Science and Engineering, Tongji University, \\ Shanghai 201804, People's Republic of China
}

(Received 26 May 2017; revised manuscript received 4 September 2017; published 19 January 2018)

\begin{abstract}
Shear transformations, as fundamental rearrangement events operating in local regions, hold the key of plastic flow of amorphous solids. Despite their importance, the dynamic features of shear transformations are far from clear, which is the focus of the present study. Here, we use a colloidal glass under shear as the prototype to directly observe the shear-transformation events in real space. By tracing the colloidal-particle rearrangements, we quantitatively determine two basic properties of shear transformations: local shear strain and dilatation (or free volume). It is revealed that the local free volume undergoes a significantly temporary increase prior to shear transformations, eventually leading to a jump of local shear strain. We clearly demonstrate that shear transformations have no memory of the initial free volume of local regions. Instead, their emergence strongly depends on the dilatancy ability of these local regions, i.e., the dynamic creation of free volume. More specifically, the particles processing the high dilatancy ability directly participate in subsequent shear transformations. These results experimentally enrich Argon's statement about the dilatancy nature of shear transformations and also shed insight into the structural origin of amorphous plasticity.
\end{abstract}

DOI: 10.1103/PhysRevApplied.9.014023

\section{INTRODUCTION}

Understanding plasticity, i.e., how solids flow, is a classical problem but still remains open. The plasticity of crystals has been well described in terms of dislocation mobility, deformation twining, grain-boundary diffusion, etc. These classical pictures, however, break down in the face of amorphous solids lacking long-range period order. Instead, it is generally recognized that amorphous plasticity results from the accumulation of local irreversible rearrangements occurring within "zones" a few to hundreds of atoms or particles wide. Such rearrangement events were originally defined as "shear transformations (STs)" by Argon in 1979 [1] that operate validly in various amorphous solids ranging from colloidal glasses and granular media to metallic glasses. Usually, avalanches of STs lead to a catastrophic failure of amorphous solids via shear

\footnotetext{
yunzhuohit@gmail.com

†mqjiang@imech.ac.cn

‡yjhuang@hit.edu.cn
}

localization [2-3]. It is therefore crucial to understand the basic properties of dynamic STs.

In the past decades, great efforts have been made to capture and theorize the ST events. Some landmark works must be highlighted. The atomistic scenario of STs was first observed by Falk and Langer in 1998 based on moleculardynamics simulations [4]. Furthermore, they developed the shear-transformation-zone (STZ) theory [4-6] of amorphous plasticity, where the populations of two-state STZs enter the flow equation as order parameters. Associating STs with relaxations on a potential energy landscape, Johnson and Samwer [7] proposed a cooperative shear model, describing how isolated STs confined within the elastic matrix develop into macroscopic plastic yielding [8-9]. Such a physical picture has been confirmed by atomistic simulations [2,10-11], continuum modeling [12-13], and direct colloidal-glass visualization [3,14-15]. As a result, the fundamental properties, i.e., activation barriers [16-17], characteristic sizes [9,18], and transformation fields [11,19], of STs can be identified quantitatively.

It is broadly accepted that STs are transient in time, giving rise spatially to Eshelby fields around them. They do 
not preexist in a glassy structure but are a consequence of complex thermally activated structural rearrangements driven by an applied shear stress. Nevertheless, it is not suggested that the STs are independent of the structures. For example, it is widely believed that STs occur preferably at sites of high free volume [1,20-21]. Recently, a number of physical parameters have been proposed to predict the structural origin of STs, including a low-frequency vibrational mode [22-23], local fivefold symmetry [24], a flow unit [25], local yield stress [26], local potential energy [27], flexibility volume [28], local thermal energy [29], etc. These parameters hold more or less predictive power but, to a great extent, from a spatiotemporally statistical view. Building a deterministic one-to-one correlation between dynamics STs and static structures still remains a formidable challenge.

It is not the purpose of the present work to directly explore the structural birth of STs. In contrast, we focus on the dynamic process of STs themselves due to their transient nature. The ST dynamics should provide an integrated picture of ST, from its birth to finality. Furthermore, it is interesting to experimentally trace the dynamic evolution of free volume during STs, which has never been touched upon so far. A long-standing idea is that free volume, as a basic structural indicator, is of paramount importance in promoting STs [1,20-21]. But recent works indicate that there seems no strong correlation between particles having high free volume and those undergoing STs $[26,30]$. Here we attempt to offer an experimental judgment for this disagreement. More importantly, the free volume dynamics during STs could reflect another fundamental property of STs, i.e., the dilatancy. On the basis of an atomic-analog 2D bubble-raft model [31], Argon stated that the net effect of the STs is an excess dilatation that must create at least temporarily additional excess free volume [1]. Recently, based on the interaction of STs and ST-mediated free volume dynamics, a constitutive theory of amorphous plasticity was proposed [13]. It indeed predicts that, for glasses with a typical strain-softening feature, STs always produce free volume, indicating their dilatancy nature. To our best knowledge, however, there has been no experimental scrutiny of the dilatancy of STs in the perspective of a real 3D amorphous solid.

In this work, we employ a high-speed confocal microscopy to directly track the motion of individual particles in a 3D colloidal glass that is subject to a shear-loading and -unloading cycle. We accurately identify where and when the ST events take place by visualizing the spatiotemporal evolution of local shear strain of particles. Both the static free volume prior to STs and its subsequent evolution within STs are also examined. It demonstrates that the emerging STs are weakly correlated with the initial free volume, even though the latter is spatially averaged over the secondneighbor shell, an optimum length. Nevertheless, the activations of STs prefer these regions with the high-dilatancy capability to create sufficient free volume above a critical value. These experimental results provide a clear picture about the interplay of STs and free volume dynamics, suggesting the dilatancy of STs or, in other words, the catalytic effect of dynamic (not static) free volume in STs.

\section{EXPERIMENTAL}

We use $1.55-\mu \mathrm{m}$-diameter silica particles with a polydispersity smaller than $3.5 \%$ to prepare a colloidal glass. The silica particles are suspended in a mixture (solvent) of deionized water (30\% vol) and dimethylsulfoxide (70\% vol). The excellent index matching between the particles and solvent allows us to take optical images deep into the colloidal system with minimal light loss due to scattering. To make the particles appear as dark spots on a bright background under fluorescence microscopy, we dye them with a fluorescein- $\mathrm{NaOH}$ solution. The density $\left(2.0 \mathrm{~g} / \mathrm{cm}^{3}\right)$ of the suspended particles is nearly twice larger than that of the solvent, with the density difference of about $\Delta \rho \approx 0.9 \mathrm{~g} / \mathrm{cm}^{3}$. Because of this density difference, the colloidal glassy state can be achieved via spontaneous sedimentation under gravity. The Péclet number Pe for the sedimentation at room temperature $T$ under gravity is

$$
\mathrm{Pe}=\frac{\Delta \rho g R^{4}}{k_{B} T} \approx 0.8
$$

where $g$ is the gravity constant, $R$ is the radius of the colloidal particles, and $k_{B}$ is the Boltzmann constant. The sedimentation starts with an initial deposition flux $\phi_{0} \mathrm{Pe} \approx 0.032$, where $\phi_{0} \approx 4 \%$ is the initial volume fraction of the particles. This deposition flux corresponds to a sufficiently high quenching rate, guaranteeing the glass transition [32]. Finally, we obtain a colloidal glass with the volume fraction of roughly 0.61 .

A shear-loading and -unloading cycle is applied to the colloidal glass. The experimental apparatus is the same as that described by Jensen, Weitz, and Spaepen [15]. As illustrated in Fig. 1(a), we carefully introduce a transmission electron microscope (TEM) grid deep into the glass. Through a hollow post, we use a piezoelectric translation stage to move the grid to apply a shear and subsequent reverse shear along the $y$ direction at a very small rate of about $4 \times 10^{-5} \mathrm{~s}^{-1}$. In the $z$ direction, highspeed confocal microscopy is utilized to visualize individual particles in a $77 \times 77 \times 27 \mu \mathrm{m}^{3}$ observation volume that is far away from the boundaries. Thus, the particle positions in $3 \mathrm{D}$ space can be determined. We track the trajectories of individual particles for the 4200-s duration of each cycle by acquiring 3D image stacks every $200 \mathrm{~s}$. Each image stack takes $200 \mathrm{~s}$.

In order to identify where and when STs occur in this sheared glass, we examine the local shear strain $\varepsilon_{i j}$ of each 


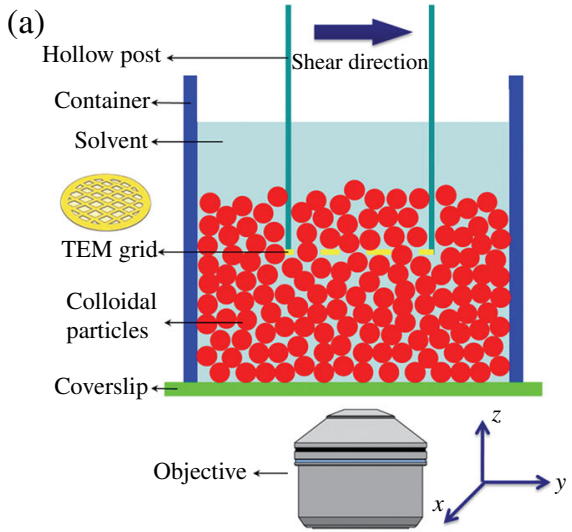

(b)
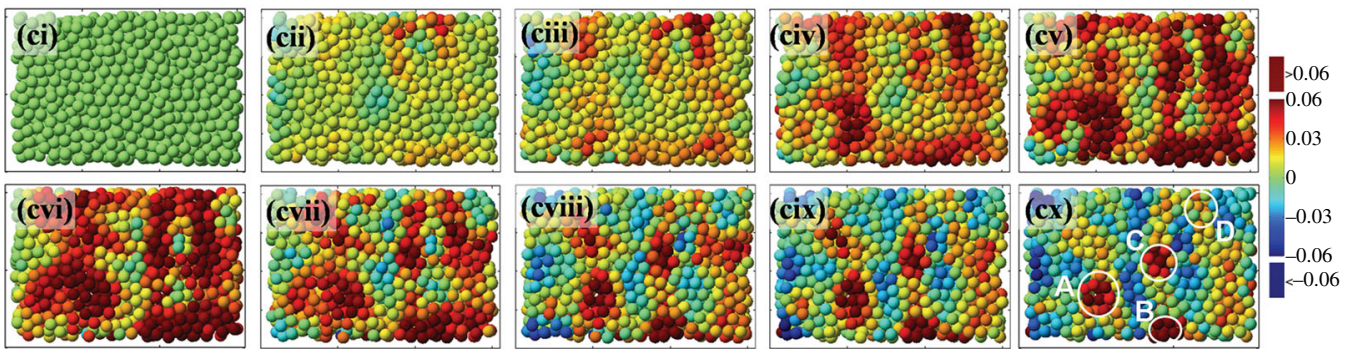

FIG. 1. A colloidal glass under a shear-loading and -unloading cycle. (a) Schematic showing the experimental setup. (b) Macroscopic shear strain applied on the colloidal glass. (ci)-(cx) $\mathrm{Cu}-$ mulative distribution of local shear strain $\varepsilon_{y z}$ of individual particles in 6- $\mu$ m-thick $y-z$ sections centered at $x=24 \mu \mathrm{m}$, corresponding to the times (i)-(x), respectively, marked in (b). particle [33]. The local shear strain $\varepsilon_{i j}$ is calculated from the relative motion of a particle, whose position is $\mathbf{r}(t)$ at arbitrary time $t$, with respect to its nearest neighbors $n$. The nearest neighbors can be determined according to the radial distribution function (RDF). We first define the nearestneighbor vectors $\mathbf{d}_{n}(t)=\mathbf{r}(t)-\mathbf{r}_{n}(t)$. Then the best affine transformation tensor $\Gamma$ that transforms the change of $\mathbf{d}_{n}(t)$ over the time interval $\Delta t$ can be determined by minimizing the mean-square difference [4] $\sum_{n}\left[\mathbf{d}_{n}(t+\Delta t)-\Gamma \times\right.$ $\left.\mathbf{d}_{n}(t)\right]^{2}$. The local shear strain $\varepsilon_{i j}$ is defined as the symmetric part of the best affine deformation tensor $\boldsymbol{\Gamma}$ [14]. The macroscopic shear strain $2\left\langle\varepsilon_{i j}\right\rangle$ is the double average of $\varepsilon_{i j}$ for all particles, and the evolution of $2\left\langle\varepsilon_{i j}\right\rangle$ during the shear cycle is shown in Fig. 1(b). As we designed that, the contributions to the macroscopic shear strain come mainly from the component $2\left\langle\varepsilon_{y z}\right\rangle$, and other components $2\left\langle\varepsilon_{x y}\right\rangle$ and $2\left\langle\varepsilon_{z x}\right\rangle$ are negligibly small. The $2\left\langle\varepsilon_{y z}\right\rangle$ increases linearly from zero to a maximum of about 0.076 at $4 \times 10^{-5} \mathrm{~s}^{-1}$ and then returns to zero at the same rate. Thus, the local shear strain $\varepsilon_{y z}$ is adopted to monitor STs in the present colloidal glass.

To explore the dilatancy of STs, we calculate the particle free volume $v_{f}[33]$ that is believed to be induced by the shear-dilatation effect [34-35]. As originally defined by Turnbull and Cohen [36], the free volume represents the space within which the center of a particle can move without changing local energy or moving its neighbors. First, we construct Voronoi polyhedra that include all points closer to the particle than to any other particle. We then move the Voronoi faces inwards by a distance of the particle diameter. The remaining space gives an estimation of the free volume $[30,37]$.

\section{RESULTS AND DISCUSSION}

The spatial distribution of the cumulative $\varepsilon_{y z}$ values with the reference zero-strain state in $6-\mu$ m-thick $y-z$ sections centered at $x=24 \mu \mathrm{m}$ is shown in Figs. 1(ci)-1(cx), corresponding to the times (i)-(x) marked in Fig. 1(b). With the shear loading, an increasing number of particles participate in shear deformation but with a spatially inhomogeneous feature. Some localized regions with large $\varepsilon_{y z}$ are emerging and eventually coalesce at the end of loading. But, in fact, not all of these localized regions experience ST events. Here, the shear strain consists of elastic (reversible) and inelastic or plastic (irreversible) parts. Only STs contribute to the plastic part, although they are elastic coupling $[19,30]$. To exactly ferret out the localized regions where STs take place, it is necessary to release the elastic part of the shear strain and just retain the plastic part. To this end, we slowly reverse shear on the glass back to zero macroscopic strain. After the elastic unloading, most particles return to their initial positions with a very low strain state. We can readily identify three surviving localized regions, marked by circles $A, B$, and $C$, respectively, in Fig. 1(cx), that have a relatively high plastic strain. It is reasonably believed that these localized regions experience irreversible STs during the shear deformation.

Next, we explore when the ST events take place in these localized regions. The ST is essentially a local cluster of atoms that undergoes an inelastic shear distortion from one relatively low-energy configuration to a second such configuration. This huge structural modification accommodates a great change in the local shear strain. Thus, we can use a local strain mutation to probe the occurrence of ST events. We then calculate the evolution of the average 


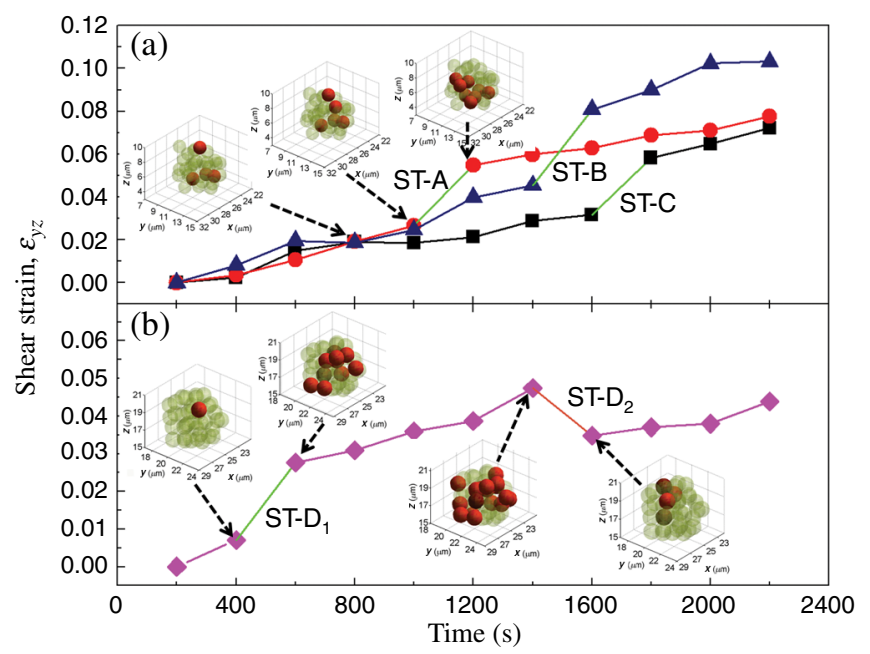

FIG. 2. Evolution of the shear strain in localized regions with shear transformations labeled as (a) ST-A, ST-B, and ST-C, and (b) ST-D from the initial time to $2200 \mathrm{~s}$. The insets show the reconstructed 3D pictures of structural rearrangements accompanied with the shear transformations.

shear strain $\varepsilon_{y z}$ of the regions $A, B$, and $C$, from the initial time to shear ending (2200 s). As presented in Fig. 2(a), the average shear strain $\varepsilon_{y z}$ of region $A$ slowly increases during the initial $1000 \mathrm{~s}$. After that, a large strain jump $\Delta \varepsilon_{y z}$, marked by a green line, occurs during 1000-1200 s, indicating the operation of a ST event (labeled as ST-A hereafter). This ST-A event should be underpinned by a significant structural rearrangement that can be indicated by the variation in the number of nearest neighbors of each particle in region $A$ [33]. We reconstruct typical 3D pictures of particle positions of region $A$ at 800,1000 , and $1200 \mathrm{~s}$, respectively, as shown in the insets in Fig. 2(a). The red solid spheres in these insets represent those particles that lose three or more nearest neighbors with the reference zero-strain state. Other particles are shown transparently to improve visibility. Comparing the reconstructed 3D pictures at 1000 and $1200 \mathrm{~s}$, the ST-A event indeed involves a large change of neighbors, corresponding to a pronounced rearrangement of particles. Similar behaviors, including both strain jumps and structural rearrangements, are also observed in regions $B$ and $C$, where the $\mathrm{ST}$ events (labeled as ST-B and ST-C, respectively) occur during 1400-1600 and $1600-1800 \mathrm{~s}$, respectively.

Falk and Langer $[4,38]$ propose that potential ST regions are two-state systems. In the presence of a shear stress, STs can operate along an either shear-positive or -negative direction. Obviously, regions $A, B$, and $C$ undergo only positive ST events that contribute to a sudden increase of the local plastic strain [Fig. 2(a)]. It is expected that the negative ST events, analogous to shear-assisted relaxation, will eliminate the local plastic strain. A localized ST region may show a relatively low plastic strain, if both positive and negative STs occur within it. Therefore, such ST regions may be hidden by their local plastic strain. To uncover them, we carefully examine the evolution of local shear strain of all particles in the glass section [Fig. 1(cx)] from the initial time to $2200 \mathrm{~s}$. We indeed find a hidden ST region, marked by the circle " $D$ " in Fig. 1(cx), where two ST events (labeled as ST- $\mathrm{D}_{1}$ and ST- $\mathrm{D}_{2}$, respectively) with opposite directions take place. The evolution of the average shear strain $\varepsilon_{y z}$ of this region is presented in Fig. 2(b). The ST- $\mathrm{D}_{1}$ event, indicated by the first large strain jump (the green line), occurs during 400-600 s. The ST- $\mathrm{D}_{2}$ event, indicated by the second large strain jump (the orange line), occurs during 1400-1600 s. It is found that the ST- $\mathrm{D}_{1}$ induced strain partly recovers due to the activation of ST- $D_{2}$. Again, both ST- $\mathrm{D}_{1}$ and ST- $\mathrm{D}_{2}$ correspond to the significant rearrangements of particles in region $D$, as indicated by the reconstructed 3D pictures shown in the insets in Fig. 2(b).

In order to quantify the correlation between the initial free volume and subsequent STs, we define a normalized correlation coefficient:

$$
C_{\varepsilon_{y z}, v_{f}}=\frac{\sum_{k}\left(\varepsilon_{y z, k}-\left\langle\varepsilon_{y z}\right\rangle\right)\left(v_{f, k}-\left\langle v_{f}\right\rangle\right)}{\sqrt{\sum_{k}\left(\varepsilon_{y z, k}-\left\langle\varepsilon_{y z}\right\rangle\right)^{2} \sum_{k}\left(v_{f, k}-\left\langle v_{f}\right\rangle\right)^{2}}},
$$

where $k$ denotes a particle that participates in the ST events, $\varepsilon_{y z, k}$ is the local shear strain of such a particle $k$ at $4200 \mathrm{~s}$, $v_{f, k}$ is its initial free volume, and $\left\langle\varepsilon_{y z}\right\rangle$ and $\left\langle v_{f}\right\rangle$ are their average values of all particles involved in STs, respectively. According to Fig. 2, the positive-strain jumps $\Delta \varepsilon_{y z}$ for all ST events are greater than a critical $\Delta \varepsilon_{y z}^{*} \sim 0.021$. Therefore, the particles that undergo STs can be identified as those with $\Delta \varepsilon_{y z} \geq \Delta \varepsilon_{y z}^{*}$ during one time step (200 s). The calculated $C_{\varepsilon_{y z}, v_{f}}$ is only about 0.24 , implying a weak link between the initial free volume and the subsequent ST events. Since STs usually occur within a length scale beyond the nearest-neighbor shell, the coarse-grained free volume may show a better correlation with STs. Here we coarse-grain the initial $v_{f, k}$ by spatially averaging over a particle and its surrounding particles lying within a coarsegraining radius $d$. As shown Fig. 3(a), with increasing $d$, the calculated $C_{\varepsilon_{y z}, v_{f}}$ increases dramatically from the initial 0.24 to a peak of 0.41 and then decreases gradually. It is interesting to find that the optimum $d \approx 3 \mu \mathrm{m}$ corresponding to the $C_{\varepsilon_{y z}, v_{f}}$ peak is very close to the second-neighbor shell of the present colloidal glass. This is confirmed by the RDF shown in the inset in Fig. 3(a). Although the $C_{\varepsilon_{y z}, v_{f}}$ is improved, the coarse-grained initial free volume still does not show a strong correlation with the ST events. This can be further confirmed from Fig. 3(b), that presents the distribution of the initial free volume after the optimum coarse-graining in the same section of Fig. 1(c). The particles $\left(\Delta \varepsilon_{y z} \geq \Delta \varepsilon_{y z}^{*}\right)$ that experience ST-A, ST-B, ST-C, and ST-D events are superimposed as marked by small 
(a)

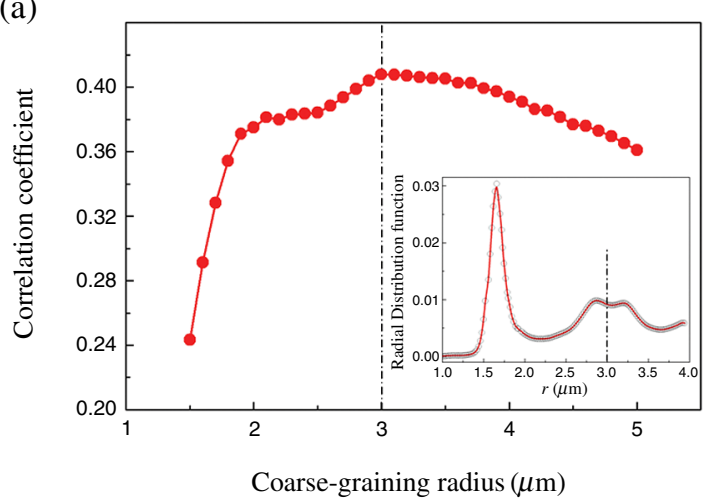

(c)

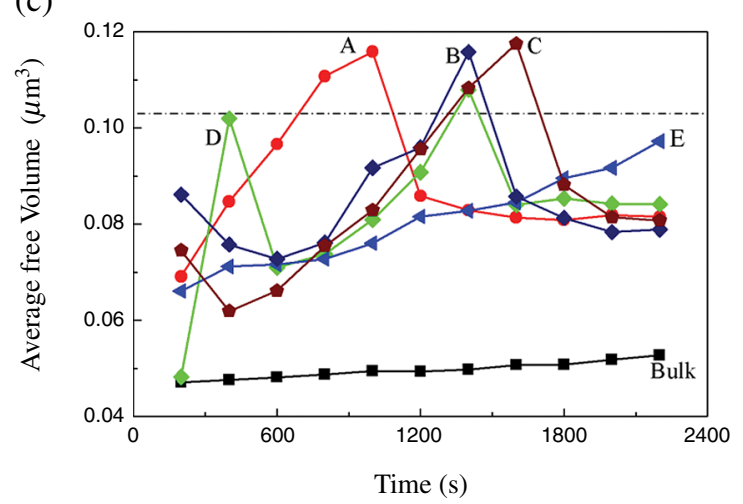

(b)

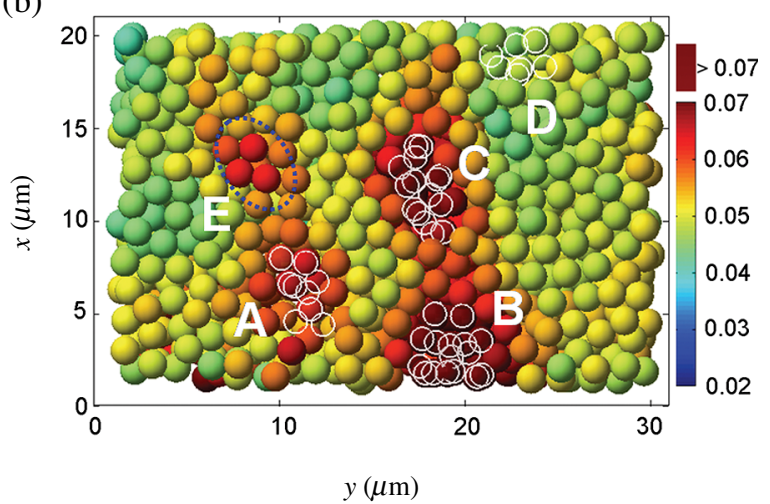

(d)

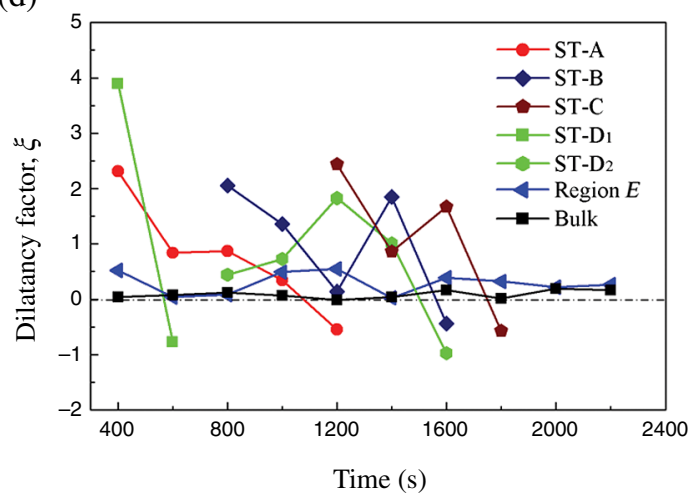

FIG. 3. Correlation between shear transformations and free volumes. (a) Normalized correlation coefficient between local shear strains of particles participating in shear transformations and their initial free volumes as a function of the coarse-graining radius. The inset shows the radial distribution function of the colloidal glass. (b) Spatial distribution of the initial free volume after the optimum coarsegraining in the same section of Fig. 1(c). Small white circles denote the particles participated in shear transformations. Dynamic evolution of (c) average free volumes and (d) dilatancy factors in typical local regions $A-E$ in (b) and the entire bulk glass from the initial time to $2200 \mathrm{~s}$.

white circles. We indeed find that not all ST events take place in the high free volume regions. In contrast to the ST-A, ST-B, and ST-C events at high free volume regions, the ST-D region has a very low free volume. Furthermore, region $E$ with a relatively high free volume does not undergo ST events, establishing that the high static free volume is not a necessary precondition for the emergence of STs.

We further examine the free volume dynamics associated with STs. We calculate the evolution of average free volume in the four localized regions $(A-D)$ with STs and the one $(E)$ without STs from initial to $2200 \mathrm{~s}$. The average free volume of the entirely bulk glass is also included for comparison. All results are shown in Fig. 3(c). The averaged bulk free volume increases slightly during the whole shear deformation. In region $A$, the local free volume first increases from the initial $0.069 \mu \mathrm{m}^{3}$ to a maximum of approximately $0.116 \mu \mathrm{m}^{3}$ due to the shear-induced dilatation. Immediately after that, the ST-A event occurs and the accompanied significant rearrangement leads to an instantaneous loss of approximately $27 \%$ of the free volume. Eventually, the free volume tends towards a steady-state value of approximately $0.082 \mu \mathrm{m}^{3}$ due to a dynamic balance between dilatation and relaxation. In regions $B$ and $C$, the relatively higher free volume first shows a decrease via relaxation then followed by an increase to a peak before the occurrence of the ST event rather than a direct increase like that in region $A$. This implies that the STs do not seem to have a memory of their initial free volume. Again, after the STs, a steady-state free volume is achieved in either region $B$ or $C$. In region $D$, where two ST events take place, the initial free volume is small, almost equal to the averaged bulk value. However, such a small initial free volume does not hinder the following ST events. Because of the significant dilatation effect, the local free volume of this region rapidly increases to a very large value of about $0.1 \mu \mathrm{m}^{3}$, which fertilizes the first $\mathrm{ST}^{-\mathrm{D}_{1}}$ event. Accompanied with the ST- $\mathrm{D}_{1}$, the free volume abruptly decreases to a smaller value. Afterwards, the free volume reincreases to the $0.11-\mu \mathrm{m}^{3}$ level, resulting in the second ST- $\mathrm{D}_{2}$ event, and eventually the free volume decreases towards a steady-state value. Eliminating the effect of initial low-strain relaxation, the local free volume of the ST regions always increases albeit the partial decrease due 
to STs. From the dynamic evolution of local free volume in regions $A, B, C$, and $D$, we notice that a significant free volume creation to a critical value $v_{f}^{*}$ is necessary for the activation of STs. Interestingly, it seems that this critical $v_{f}^{*}$ has no direct link with the initial value. We calculate the $v_{f}^{*}$ by averaging the maximum free volume of all ST particles just before ST events take place. Then the critical free volume $v_{f}^{*}$ for STs in our colloidal glass is about $0.103 \mu \mathrm{m}^{3}$, signed by a dot-dashed line in Fig. 3(c). Obviously, in region $E$, although its initial value is relatively large, the free volume is always lower than the $v_{f}^{*}$ during the whole shear process. Thus, no ST events occur in this region.

It can be seen from Fig. 3(c) that the main difference in the free volume evolution among regions with and without STs is the dynamic creation of free volume. In regions $A-D$ with STs, the local free volume can rapidly increase upon the critical $v_{f}^{*}$ for preparing the ST operations. But in region $E$ without STs, the free volume increases slowly and never reaches the critical $v_{f}^{*}$. These results indicate that the creation ability of free volume is pivotal for controlling the activation of STs. In order to measure such a creation ability, we define a dilatancy factor for each particle with its volume of $v_{0}$ :

$$
\xi=\frac{\Delta v_{f} / v_{0}}{\left|\Delta \varepsilon_{y z}\right|},
$$

which evaluates the increase $\Delta v_{f}$ of free volume induced by a shear strain increment $\Delta \varepsilon_{y z}$ during one time step (200 s). Figure 3(d) gives the calculated results about the evolution of average dilatancy factors in regions $A-E$ and the bulk glass. Clearly, for these ST regions $A-D$, the average $\xi$ are all very large before the operation of ST events and usually higher than 1 . Very interestingly, Argon defined a similar ST dilatancy $\bar{\beta}$ and also found $\bar{\beta}=1$ for shear amorphous soap-bubble rafts [39-40]. Region $E$ without STs shows only a relatively low $\xi$ with the maximum of about 0.35 over the whole period. Since the STs highly localize into only the four regions, the average bulk $\xi$ also keeps a low level less than 0.2. Thus, we have a conclusion that the ST events preferentially occur in those regions with the high-dilatancy ability of creating free volume, rather than those with the initial high free volume. To prove this more directly, we construct a glass section corresponding to that of Fig. 1(cx) in Fig. 4. The particles with the free volume $v_{f}^{m}$ larger than $0.09 \mu \mathrm{m}^{3}$ are presented as solid spheres. Other particles are displayed transparently to improve visibility. Here, $v_{f}^{m}$ is the highest free volume of the particle during the whole shear process during the initial to $2200 \mathrm{~s}$. The particle color denotes the dilatancy factor $\xi$ of the particle that is averaged over three adjacent time steps (600 s) prior to reaching the largest free

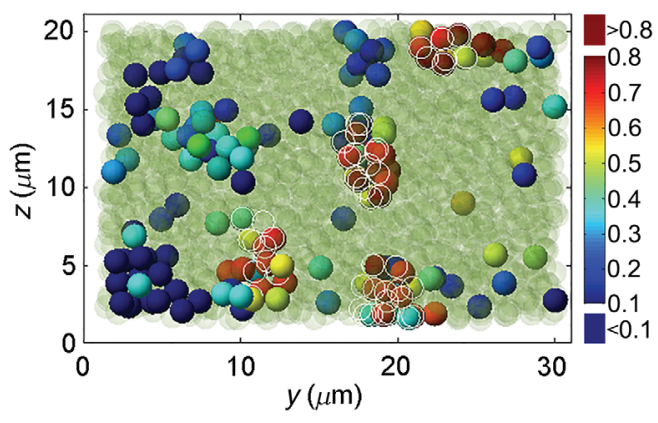

FIG. 4. Spatial distribution of dilatancy factors of particles with high free volume (solid spheres), superimposed upon particles participating in shear transformations (white circles).

volume $v_{f}^{m}$. The particles that undergo the ST events are again marked by small white circles. We find that there are many particles that have processed a large free volume at certain shear times. However, not all of them experience ST events; instead, only those having the high-dilatancy factor contribute to STs. An excellent correlation can be observed between them, experimentally supporting that the dilatancy is an inherent nature of ST events in glasses.

\section{CONCLUSIONS}

The main purpose of the present study is to experimentally probe the free volume dynamics associated with STs and qualitatively analyze their relationship between the two dynamic processes. These aspects are original, not covered in previous reports [14-15,30]. For this purpose, we perform an in situ observation of the dynamic ST events in a 3D colloidal glass under a simple shear deformation and meanwhile trace the spatiotemporal evolution of free volume in these local ST regions. An intriguing finding is that the dilatancy is an inherent nature of transient STs, supporting, but beyond, Argon's statement [1]. This is embodied by the following two aspects. First, the occurrence of STs prefer the local regions having high-dilatancy ability of creating sufficient free volume, where their initial, static free volume is not necessarily high. Second, the local free volume of these ST regions eventually increases to a steady-state value albeit the existence of a temporal, partial decrease due to STs. Although its precise picture is not very clear, the dilatancy of STs might result from the complex interaction between the ST regions and their neighborhood, closely depending on the local free volume, temperature, and stress fields. These results, for the experimental viewpoint, bridge a gap between the free volume dynamics and the ST operations, which is a crucial step for developing a general theory of amorphous plasticity $[13,21,35,41]$, as proposed by Spaepen in his "flow defect" concept [42]. The present work also confirms that the thermally activated ST theory for amorphous plasticity may extend to colloidal glasses [14-15,30]. At last, our findings suggest that, for amorphous media, the prediction or prevention of their 
mechanical failure may need a real-time and in situ monitor into the dynamic evolution of structural defects [43-44]. This demands the development of advanced experimental apparatus and technology.

\section{ACKNOWLEDGMENTS}

This work was supported by the National Natural Science Foundation of China (Grants No. 11522221, No. 51401041, No. 11372315, No. 51671042, No. 51671070, and No. 51274151), the Strategic Priority Research Program of the Chinese Academy of Sciences (Grant No. XDB22040303), the China Postdoctoral Science Foundation (Grant No. 2015M570242), and the Basic Research Program of the Key Lab in Liaoning Province Educational Department (Grant No. LZ2015011). The first experiments leading to this work were made during Y. Z. L.'s stay in F. Spaepen's and D. A. Weitz's laboratories at Harvard University. He thanks Katharine E. Jensen and J. Zsolt Terdik for their advice and assistance.

[1] A. S. Argon, Plastic deformation in metallic glasses, Acta Metall. 27, 47 (1979).

[2] A. Lemaítre and C. Caroli, Rate-Dependent Avalanche Size in Athermally Sheared Amorphous Solids, Phys. Rev. Lett. 103, 065501 (2009).

[3] V. Chikkadi, G. Wegdam, D. Bonn, B. Nienhuis, and P. Schall, Long-Range Strain Correlations in Sheared Colloidal Glasses, Phys. Rev. Lett. 107, 198303 (2011).

[4] M. L. Falk and J.S. Langer, Dynamics of viscoplastic deformation in amorphous solids, Phys. Rev. E 57, 7192 (1998).

[5] L. O. Eastgate, J. S. Langer, and L. Pechenik, Dynamics of Large-Scale Plastic Deformation and the Necking Instability in Amorphous Solids, Phys. Rev. Lett. 90, 045506 (2003).

[6] E. Bouchbinder and J. S. Langer, Linear Response Theory for Hard and Soft Glassy Materials, Phys. Rev. Lett. 106, 148301 (2011).

[7] W. L. Johnson and K. Samwer, A Universal Criterion for Plastic Yielding of Metallic Glasses with a $\left(\mathrm{T} / \mathrm{T}_{g}\right)^{2 / 3}$ Temperature Dependence, Phys. Rev. Lett. 95, 195501 (2005).

[8] J. S. Harmon, M. D. Demetriou, W. L. Johnson, and K. Samwer, Anelastic to Plastic Transition in Metallic GlassForming Liquids, Phys. Rev. Lett. 99, 135502 (2007).

[9] M. Zink, K. Samwer, W. L. Johnson, and S. G. Mayr, Plastic deformation of metallic glasses: Size of shear transformation zones from molecular dynamics simulations, Phys. Rev. B 73, 172203 (2006).

[10] Y. F. Shi and M. L. Falk, Strain Localization and Percolation of Stable Structure in Amorphous Solids, Phys. Rev. Lett. 95, 095502 (2005).

[11] T. Albaret, A. Tanguy, F. Boioli, and D. Rodney, Mapping between atomistic simulations and Eshelby inclusions in the shear deformation of an amorphous silicon model, Phys. Rev. E 93, 053002 (2016).
[12] L. Li, E. R. Homer, and C. A. Schuh, Shear transformation zone dynamics model for metallic glasses incorporating free volume as a state variable, Acta Mater. 61, 3347 (2013).

[13] M. Q. Jiang, G. Wilde, and L. H. Dai, Origin of stress overshoot in amorphous solids, Mech. Mater. 81, 72 (2015).

[14] P. Schall, D. A. Weitz, and F. Spaepen, Structural rearrangements that govern flow in colloidal glasses, Science $\mathbf{3 1 8}$, 1895 (2007).

[15] K. E. Jensen, D. A. Weitz, and F. Spaepen, Local shear transformations in deformed and quiescent hard-sphere colloidal glasses, Phys. Rev. E 90, 042305 (2014).

[16] S. G. Mayr, Activation Energy of Shear Transformation Zones: A Key for Understanding Rheology of Glasses and Liquids, Phys. Rev. Lett. 97, 195501 (2006).

[17] H. B. Yu, X. Shen, Z. Wang, L. Gu, W. H. Wang, and H. Y. Bai, Tensile Plasticity in Metallic Glasses with Pronounced $\beta$ Relaxations, Phys. Rev. Lett. 108, 015504 (2012).

[18] D. Pan, A. Inoue, T. Sakurai, and M. W. Chen, Experimental characterization of shear transformation zones for plastic flow of bulk metallic glasses, Proc. Natl. Acad. Sci. U.S.A. 105, 14769 (2008).

[19] J. Chattoraj and A. Lemaître, Elastic Signature of Flow Events in Supercooled Liquids Under Shear, Phys. Rev. Lett. 111, 066001 (2013).

[20] C. A. Schuh, T. C. Hufnagel, and U. Ramamurty, Mechanical behavior of amorphous alloys, Acta Mater. 55, 4067 (2007).

[21] A. Lemaítre, Rearrangements and Dilatancy for Sheared Dense Materials, Phys. Rev. Lett. 89, 195503 (2002).

[22] M. L. Manning and A. J. Liu, Vibrational Modes Identify Soft Spots in a Sheared Disordered Packing, Phys. Rev. Lett. 107, 108302 (2011).

[23] J. Ding, S. Patinet, M. L. Falk, Y. Q. Cheng, and E. Ma, Soft spots and their structural signature in a metallic glass, Proc. Natl. Acad. Sci. U.S.A. 111, 14052 (2014).

[24] H. L. Peng, M. Z. Li, and W. H. Wang, Structural Signature of Plastic Deformation in Metallic Glasses, Phys. Rev. Lett. 106, 135503 (2011).

[25] Z. Lu, W. Jiao, W. H. Wang, and H. Y. Bai, Flow Unit Perspective on Room Temperature Homogeneous Plastic Deformation in Metallic Glasses, Phys. Rev. Lett. 113, 045501 (2014).

[26] S. Patinet, D. Vandembroucq, and M. L. Falk, Connecting Local Yield Stresses with Plastic Activity in Amorphous Solids, Phys. Rev. Lett. 117, 045501 (2016).

[27] Y. Shi, M. B. Katz, H. Li, and M. L. Falk, Evaluation of the Disorder Temperature and Free-Volume Formalisms via Simulations of Shear Banding in Amorphous Solids, Phys. Rev. Lett. 98, 185505 (2007).

[28] J. Ding, Y.-Q. Cheng, H. Sheng, M. Asta, R. O. Ritchie, and E. Ma, Universal structural parameter to quantitatively predict metallic glass properties, Nat. Commun. 7, 13733 (2016).

[29] J. Zylberg, E. Lerner, Y. Bar-Sinai, and E. Bouchbinder, Local thermal energy as a structural indicator in glasses, Proc. Natl. Acad. Sci. U.S.A. 114, 7289 (2017).

[30] Y. Rahmani, R. Koopman, D. Denisov, and P. Schall, Visualizing the strain evolution during the indentation of colloidal glasses, Phys. Rev. E 89, 012304 (2014). 
[31] A. S. Argon and H. Y. Kuo, Plastic flow in a disordered bubble raft (an analog of a metallic glass), Mater. Sci. Eng. 39, 101 (1979).

[32] K. E. Jensen, D. Pennachio, D. Recht, D. A. Weitz, and F. Spaepen, Rapid growth of large, defect-free colloidal crystals, Soft Matter 9, 320 (2013).

[33] The software for the analysis was developed by P. Schall and K. E. Jensen.

[34] F. Spaepen, A microscopic mechanism for steady state inhomogeneous flow in metallic glasses, Acta Metall. 25, 407 (1977).

[35] M. Q. Jiang, G. Wilde, and L.H. Dai, Shear band dilatation in amorphous alloys, Scr. Mater. 127, 54 (2017).

[36] D. Turnbull and M. H. Cohen, Free-volume model of the amorphous phase: Glass transition, J. Chem. Phys. 34, 120 (1961).

[37] S. Sastry, T. M. Truskett, P. G. Debenedetti, S. Torquato, and F. H. Stillinger, Free volume in the hard sphere liquid, Mol. Phys. 95, 289 (1998).
[38] J. S. Langer, Dynamics of shear-transformation zones in amorphous plasticity: Formulation in terms of an effective disorder temperature, Phys. Rev. E 70, 041502 (2004).

[39] A. S. Argon and L. T. Shi, Analysis of plastic flow in an amorphous soap bubble raft by the use of an interbubble potential, Philos. Mag. A 46, 275 (1982).

[40] A. S. Argon and L. T. Shi, Development of visco-plastic deformation in metallic glasses, Acta Metall. 31, 499 (1983).

[41] M. Q. Jiang, W. H. Wang, and L. H. Dai, Prediction of shearband thickness in metallic glass, Scr. Mater. 60, 1004 (2009).

[42] F. Spaepen, in Physics of Defects, edited by R. Balian, M. Kleman, and J. Poirier (North-Holland, Amsterdam, 1981).

[43] D. Klaumünzer, A. Lazarev, R. Maaß, F. H. Dalla Torre, A. Vinogradov, and J. F. Löffler, Probing Shear-Band Initiation in Metallic Glasses, Phys. Rev. Lett. 107, 185502 (2011).

[44] Y. Huang, J. C. Khong, T. Connolley, and J. Mi, The onset of plasticity of a Zr-based bulk metallic glass, Int. J. Plast. 60, 87 (2014). 